repressor molecules cause them to unite with one another and so become inactive as repressors. The pathway leading to clamp-connections is thus freed. Dick's hypothesis that the incompatibility factors are regulator genes is of particular interest in view of Ellingboe's discovery of a novel recombination mechanism for these genes, because there is a possibility that the more familiar recombination mechanisms of crossing-over and conversion are specific to structural genes. It is evident that study of incompatibility in fungi, in addition to its intrinsic interest, may provide fundamental information about basic genetic phenomena. This book is invaluable for all who wish to keep abreast of the remarkable advances that have been made in recent years in knowledge of fungal incompatibility.

Dr. H. L. K. Whitehouse.

\title{
PSYCHOGENETICS OF THE DOG
}

GENETICS AND THE SOCIAL BEHAVIOUR OF THE DOG. J. P. Scott and J. L. Fuller. University of Chicago Press, Chicago, 1965. Pp. $468+$ xviii. $\$ 12.50$

For years, the small world of those of us actively involved in experimental psychogenetics-or behaviour genetics as the Americans seek to designate it - have been aware of an important progamme of research on the genetics of the dog going on at the Roscoe B. Jackson Memorial Laboratory at Bar Harbor in Maine. A series of reports on various aspects of the programme in the technical journals acquainted us with its admirable scope, and our appetites were whetted by some small packets of data specifically relating to behavioural inheritance which appeared from time to time. One such was seized upon by the present reviewers, re-analysed by the methods of biometrical genetics and published by them as early as I96I. Patience has been rewarded by the publication in this book of results gathered over the I 3-year period of this considerable research project.

Scott and Fuller decided to concentrate on five representative breeds of dog-the wire-haired fox-terrier, the American cocker-spaniel, the Shetland sheepdog, the beagle and the African basenji or barkless dog. Thus most of the major group of dogs recognised by breeders were covered, except for the toy and non-sporting breeds. In addition, a programme of crossings between two of the strains, the cocker-spaniel and the basenji, was attempted and this, while not complete in terms of all the usual possibilities within the $F_{1}, F_{2}$ and backcross pattern, accounted for 201 of the 470 experimental animals reared. In principle, the intention of the authors was to subject all this experimental population to a series of specially designed psychological tests in order to assess behavioural inheritance in the dogs, rearing them in a carefully controlled and standardised fashion. The realisation of this programme and the application of it to the three breeds not involved in the cross is described, together with interesting material on the evolution of the dog, and the development, both morphological and social, of all the breeds, provide much fascinating material. The reader interested in such matters will find rewarding reading in many of the chapters and, if he is a dog owner, both he and his dog may benefit from the understanding of dog behaviour which will almost certainly follow. He should, however, be warned that Scott's comprehensive system of classification of dog behaviour into nine broad categories of behaviour, useful though it may be at a descriptive level, is idiosyncratic, especially in relation to terminology such as 
" et-epimeletic" which means relating to the solicitation of care or attention.

But it is with the kernel of this rich nut-the data emerging from the programme of cross breeding - that the readers of this journal will no doubt be primarily concerned. To what extent have Scott and Fuller succeeded in the immense task they set themselves?

Here we are concerned with matters more technical. To begin with, it was perhaps unfortunate that the complete programme of crossing was not attempted. Because all the backcrossing was done using $F_{1}$ males crossed with the relatively purebred females, and none reciprocally, one of the means of determining the basis of the reciprocal differences observed in 20 out of $5^{\circ}$ measures in the $F_{1}$ and 6 out of 50 in the $F_{2}$ was lost. The authors consider three explanations of these reciprocal effects, namely, sex linkage, maternal effects and heterogeneity of breeds, and in the absence of clear-cut evidence they favour the latter as the best overall explanation. They note, however, that the $F_{1}$ make superior mothers, and in this may lie the key to the reciprocal differences reported. But the absence of reciprocal backcrosses rules out any test of this interpretation.

As regards the important question of scaling, Scott and Fuller appreciate the need for transforming data but use as the criterion of a satisfactory scale the normality of the distribution. Normality was achieved by the use of stanine scores, a transformation often used in psychological work for normalising distributions but not necessarily the optimal one for psychogenetic purposes. No attempt has been made to minimise non-allelic interaction and genotype-environment interaction by rescaling. Their scores therefore satisfy normality irrespective of whether or not it maximises rather than minimises these interactions. No tests for these interactions were made, and, indeed, they were assumed to be absent in the interpretation of the results even though they are obviously present in many sets of data.

The assumptions the authors make are basically those of homogeneity of breeds and homogeneity of environment in interpreting variance components and in partitioning them into hereditary and environmental portions.

The authors show a preoccupation with the question of the number of genes involved in determining the phenotypes measured, and it plays an important role in their analytical approach. They use it to distinguish major genic and polygenic control. While they clearly realise that their estimates of the number of genes are minimal, they overlook the most important reason for this. It is an implicit assumption of the method of estimation used that the parents show the maximum difference in phenotype that could result from all the genes of increasing effect being present in one parent and all decreasers in the other. But even a cursory examination of some of the data for breed differences shows that this assumption cannot be met. For example, the parental breeds are intermediate, rather than extreme, in 7 out of 16 measures of emotional reactivity and in 14 out of 18 of problem solving.

When it comes to interpretation, they accept their estimates of the number of genes at face value and no longer make allowance for the underestimation. Inevitably, therefore, they conclude that few genes are responsible and in many cases that a single gene explanation will suffice. Moreover, they persist in accepting this view, even when there is no supporting evidence or even contrary indications from other aspects of the same data are present, as when, for example, the $F_{2}$ and backcross distributions show 
no evidence of the discontinuity expected from the segregation of one or a few genes of major effect.

The analysis employed displays lack of discrimination between dominance and potence, which would not matter if one accepts single gene interpretations throughout. The authors regularly determine dominance from the $F_{1}$ mean, following the unfortunate and incorrect view, which has been propagated in the behavioural literature by Bruell, that the coincidence of the $F_{1}$ mean and the mid-parent value implies no dominance, whereas this estimate of potence can be explained in several alternative and illuminating ways, which in fact invoke dominance.

Within the restrictions of possible inadequacies of scale and of their earlier assumptions, the numerous estimates of heritability which Scott and Fuller present are essentially satisfactory and establish beyond doubt the magnitude and importance of the heritable component of the behaviour studied. However, they always estimate environmental variation from the $F_{1}$ generation only, never using the comparable variances of the parents. This is unfortunate on two counts. First, it gratuitously discards half of the relevant data and, second, in the presence of unequal variances among parents and $F_{1}$ that is, when genotype-environmental interaction is present, all three must be used to give a valid estimate of environmental variation.

Despite these strictures, it is only fair to note that the authors explicitly recognise the limitations of the analytical methods they employed and realise the possibility of the application of more sophisticated methods. However, they doubt the applicability of such methods in the absence of genetic homogeneity in the parental breeds. But methods exist for dealing with such problems within the context of biometrical genetics, and it may well be that others will attempt their application to the rich collection of data on dog behaviour which Scott and Fuller have made available in this important book.

P. L. Broadhurst and J. L. Jinks.

\section{APPENDIX EXTRAORDINARY}

MENTAL RETARDATION: A FAMILY STUDY. Elizabeth W. Reed and S. C. Reed. W. B. Saunders, Philadelphia, 1965. Pp. vi+719. £7.

This book is mostly an appendix. The first 80 pages describe how the data were collected and the succeeding $65^{\circ}$ or so present them.

The research reported by Dr. Elizabeth Reed and her husband, who has been Director of the Dight Institute of Human Genetics at the University of Minnesota since I947, began as long ago as IgI I when two trained social workers began a study of the families of patients at a state institution in Minnesota. It was clearly extremely well carried out and, fortunately, intelligence tests were developed and applied early in this particular state, so that assessments of intelligence quotient are available for many of the probands in the study. In I 949 the study was reopened, and criteria carefully laid down as to which of the original families should be followed up. For example, epileptics were omitted from further consideration. The result is that no less than 80,000 people are included in this work, which is a sort of Kinsey report on mental retardation, and constitutes perhaps the most important study in this area for many years.

The authors wisely accept that, while the IQ measurements they present 